\title{
GENERALIZACIÓN DE MODELO DIGITAL DE ELEVACIÓN CONDICIONADA POR PUNTOS CRÍTICOS DE TERRENO
}

\author{
Digital Elevation Model Generalization constrained by Critical Points of the Terrain \\ CARLOS MENA FRAU \\ LEONARDO MOLINA PINO \\ YONY ORMAZÁBAL ROJAS \\ YOHANA MORALES HERNÁNDEZ \\ Universidad de Talca, Centro de Geomática. \\ Avenida Lircay s/n. Talca, Chile. \\ [cmena, yormazabal, ymorales]@utalca.cl; Imolinapino@gmail.com
}

\begin{abstract}
RESUMEN
Los Modelos Digitales de Elevación (MDE) son productos geomáticos esenciales en el estudio, manejo y administración del territorio. Su uso, así como la disponibilidad de datos de alta resolución, ha ido en crecimiento sostenido en las últimas décadas, permitiendo diversos y variados análisis cuyos resultados permiten cualificar y cuantificar los características propias del terreno. No obstante la disponibilidad creciente de datos de elevación, existen ocasiones donde estudios del territorio requieren el uso de modelos digitales de terreno con resolución espacial más gruesa que la resolución de los datos disponibles. La evaluación de un método de generalización de MDE que considera la retención de puntos críticos del terreno es abordado en el presente documento. Este método es comparado con otro método que considera solamente una interpolación bilineal aplicada al cambio de resolución del MDE. En la evaluación son utilizados datos del proyecto SRTM (Shuttle Radar Topography Mission) para una zona de $111 \mathrm{~km}$ por $91 \mathrm{~km}$ aproximadamente, en la Región del Maule, Chile. Los resultados evidencian que el método de generalización condicionada por puntos críticos presenta mejor desempeño al preservar la posición espacial de los rasgos evaluados dentro de la escala del MDE generalizado.
\end{abstract}

Palabras-clave: Generalización; Modelo Digital de Elevación; SRTM; Puntos Críticos de Terreno. 
Digital Elevation Models (DEMs) are essential geomatic products in the study, management and administration of the territory. Uses and availability of high resolution data reveal a steady growth in recent decades allowing several analyses to qualify and quantify characteristics of the territory. Despite the increasing availability of the elevation data, territories studies require DEMs with coarser spatial resolution than the available one. This report evaluates the proposed generalized DEM method which retains terrain critical-points comparing to the one that applies a bilinear interpolation to change DEM resolution. Therefore, Shuttle Radar Topography Mission (SRTM) project data of Maule Region- Chile $(111 \times 91 \mathrm{~km})$ are used in the evaluation process. Results show that the generalization constrained by critical points method has a better performance in preserving the spatial position of the evaluated features within the generalized DEM scale.

Keywords: Generalization; Digital Elevation Model; SRTM; Terrain Critical Points.

\section{INTRODUCCIÓN}

Variaciones de elevación del terreno pueden ser modeladas por diferentes métodos denominados Modelos Digitales de Elevación (MDE). Una de las variantes de los MDE son las mallas regulares, cuyo principio considera la discretización de la superficie por puntos que forman una malla de rectángulos regulares, en su proyección al plano horizontal, y donde cada punto tiene asociado un valor de elevación. Las líneas ortogonales que componen esta malla regular pueden ser consideradas como filas y columnas en un sistema matricial y por tanto, la malla puede conformar una matriz de elevación. Debido a la facilidad de la manipulación computacional de las matrices, en particular en los sistemas de información geográfica raster, las mallas de rectángulos regulares se han convertido en el modelo de elevación de mayor disponibilidad (BURROUGH y MCDONNELL, 1998). En la actualidad son muchas las fuentes de información que consideran este tipo de modelo para representar elevaciones del terreno, o incluso, usando los mismos principios, representar otras variables espaciales que puedan ser conceptualizadas como campo continuo. Con los avances tecnológicos en sensoramiento remoto, la disponibilidad de datos de elevación de terreno ha ido en aumento sostenido y cada vez con un mayor nivel de resolución espacial y precisión altimétrica (WEVER y LINDEBERGER, 1999; NIKOLAKOPOULOS et al. 2006). La misión SRTM (Shuttle Radar Topography Mission) es un ejemplo de disponibilidad de datos de elevación de acceso libre. Su cobertura espacial alcanza al 80\% del territorio terrestre, con un nivel de detalle de 30 segundos de arco, que corresponden a $90 \mathrm{~m}$ aproximadamente. Estos datos de elevación fueron obtenidos en una escala casi global para generar la más completa base digital de datos topográficos de la Tierra en alta resolución (FAR, et al. 2007).

Debido a que en un MDE de malla regular la superficie modelada se supone que es matemáticamente continua, y su modelamiento una discretización de tal superficie, es posible obtener diversos atributos del terreno, ya sea mediante el cálculo de las 
diferencias dentro de una ventana de análisis para un punto específico o mediante el ajuste de un polinomio a los datos en la ventana de análisis. Siendo así, numerosas operaciones pueden ser aplicadas obteniendo valores que tipifican los atributos de la zona representada en un MDE (BURROUGH y MCDONNELL, 1998).

Actualmente, existen métodos de análisis digital del terreno que permiten extraer desde un MDE los parámetros que caracterizan el terreno representado, como la elevación del terreno, superficie, pendiente, aspecto, curvatura y zona de influencia; clasificar características del terreno, tales como cimas, cuencas, uniones de ríos, líneas de valle y divisorias de aguas, canales, colinas y terrazas, etc. (PEUCKER y DOUGLAS, 1975; FOWLER y LITTLE, 1979; JENSON y DOMINGUE, 1988; HERRINGTON y PELLEGRINI, 2000). Por otro lado, cuando los análisis requieren el uso de una resolución espacial más gruesa, es necesario realizar procesos que permitan la generalización del MDE original a uno de menor resolución. Considerando que el uso de los MDE atinge variados objetivos y diversas aplicaciones de procesos cuantitativos, la generalización de un MDE debe considerar la mantención o preservación de los rasgos que caracterizan el terreno, con la suficiente exactitud y precisión acorde a la escala final de generalización (ZHOU y CHEN, 2010). Por esta razón la generalización cartográfica de un MDE no sólo debe considerar el aspecto geométrico o de volumen de datos, sino también, y principalmente, el mantenimiento de los rasgos y aspectos cuantitativos que caracterizan la superficie modelada (AI, 2007). Existen variados métodos de generalización de MDE que consideran estos requerimientos particulares y que incluyen en sus procesos la detección de puntos que caracterizan el terreno, con el fin de mantener los rasgos que la caracterizan (AI y LI, 2010; ZHOU y CHEN, 2010). Otro método propuesto recientemente es el presentado por HU y LIU (2009), el cual, según los autores, mantiene las propiedades y características geomorfológicas del MDE original. La propuesta metodológica puede ser resumida con la siguiente premisa: "Si en el proceso de generalización cartográfica de un MDE se mantienen los puntos con valores críticos que describen los rasgos del terreno en su contexto local, tal que estos puntos siempre se mantengan inalterados en posición horizontal y valor de elevación, entonces, el producto de la generalización del MDE mantendrá los parámetros y rasgos que caracterizan el terreno modelado”. En el trabajo presentado por HU y LIU (2009), los autores evalúan el método a través de una comparación visual del trazado entre las curvas de nivel originales, desde donde fue creado el MDE original, y las curvas de nivel obtenidas desde el MDE generalizado. En el presente documento se aborda la implementación y evaluación de este método. Se exponen los criterios para determinar puntos críticos y los criterios para incluirlos en el proceso de generalización, se aborda la evaluación del método considerando las variaciones de posición horizontal, vertical y tridimensional de puntos críticos. Estos valores son comparados con los obtenidos a partir de una generalización de MDE por cambio de resolución e interpolación por media aritmética. El método es aplicado a un MDE obtenido de los datos disponibles de la misión SRTM para una zona de 111 km por 91 km aproximadamente, en la Región 
del Maule, Chile. En la evaluación se consideraron cuatro generalizaciones a diferentes resoluciones espaciales y a partir de un MDE original de 90m de resolución espacial.

\section{METODOLOGÍA}

\subsection{Descripción de los Datos Usados en los Experimentos}

El método de generalización fue aplicado a un MDE obtenido de los datos disponibles del proyecto SRTM para una zona de 111 por $91 \mathrm{~km}$ aproximadamente, dada por una matriz de elevación de 1200 filas por 1200 columnas. Los valores de elevación están referidos a una zona de la Región del Maule, Chile, ubicada entre los paralelos $35^{\circ}$ y $36^{\circ}$ de latitud Sur, y entre los meridianos $71^{\circ} 30^{\prime}$ y $70^{\circ} 30^{\prime}$ de longitud Oeste. La zona posee un vasto sector de montaña con pendientes fuertes y una zona de valle (Figura 1). La elevación varía entre los 113m y 4.074m sobre el nivel medio del mar.

Figura 1 - Ubicación de la zona de prueba y cobertura MDE 90.

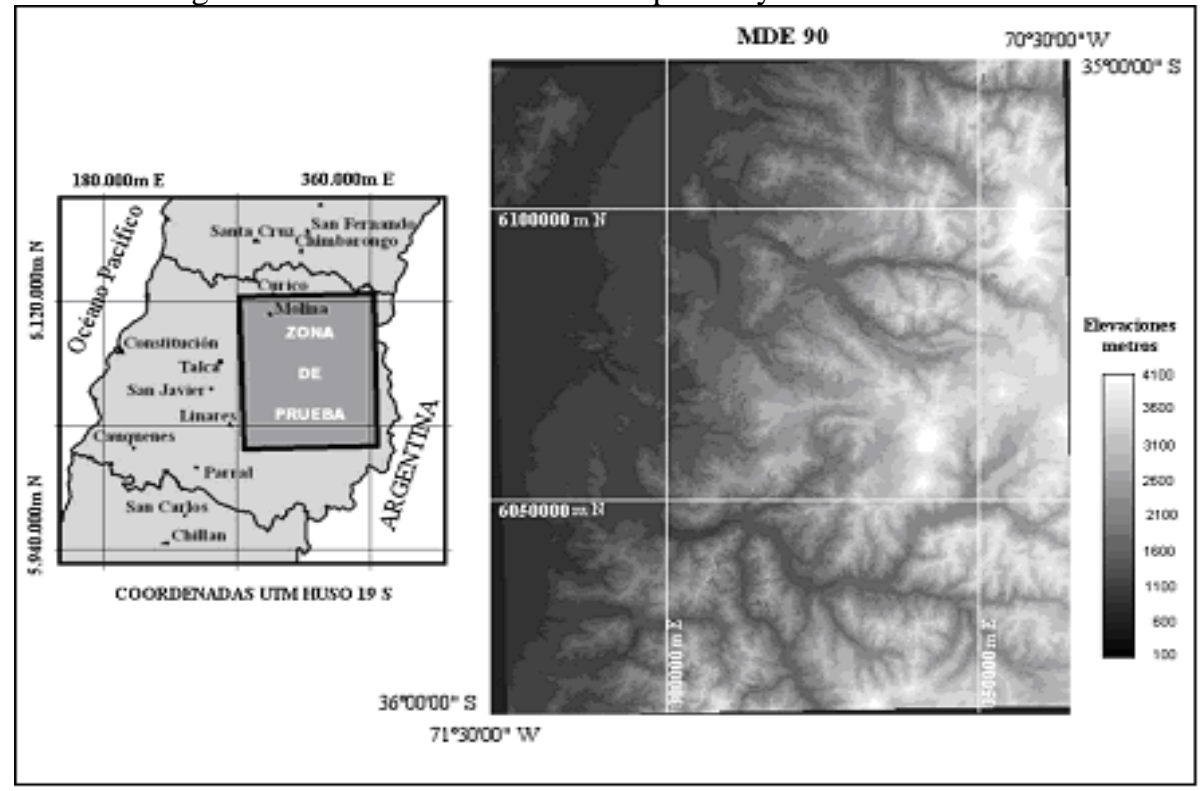

\subsection{Método de Generalización de MDE Condicionada por Puntos Críticos de Terreno.}

El método de generalización propuesto se implementa en tres etapas que corresponden a identificación de puntos críticos, aplicación de ranking de ponderación y 
generalización del MDE. La secuencia de los procedimientos puede ser observada en la figura 3 donde se expone el diagrama de flujo del método de generalización condicionada por puntos críticos.

\subsubsection{Etapa I - Identificación de Puntos con Valores Críticos Máximos y Mínimos}

En el MDE original se identifican y evalúan los puntos críticos máximos y mínimos que caracterizan el terreno. A partir de los valores de elevación contenidos en el MDE original, y por medio de operaciones de ventana de análisis, son detectados los puntos cuyos valores de elevación son críticos respecto a los valores de elevación de puntos vecinos, y por tanto, caracterizan aspectos geomofológicos del terreno representado en el MDE. Para esta aplicación los rasgos cimas y crestas denotan puntos con valores críticos máximos de elevación, mientras que la red de drenaje denota puntos con valores críticos mínimos de elevación.

\subsubsection{Etapa II - Aplicación de Ranking de Ponderación}

Se evalúa la importancia de los puntos con valores críticos respecto al valor de elevación, asignándole un valor de ranking. El procedimiento obedece a los siguientes criterios: a) los puntos con valores críticos máximos obtendrán ponderaciones en forma proporcional al valor de elevación del punto, y b) los puntos con valores críticos mínimos obtendrán ponderaciones inversamente proporcional al valor de elevación del punto. Aplicando ambos criterios, puntos asociados a cimas o crestas localizadas en sectores de mayor elevación, prevalecerán sobre puntos vecinos que describen los inicios de una red de drenaje. Así mismo, los puntos que describen la red de drenaje en zonas de menor altitud prevalecerán sobre puntos vecinos asociados a cimas o crestas. La aplicación de este ranking asegura que las altas cumbres, como así también los puntos más bajos representados en el MDE, obtendrán los valores más altos en el ranking .

\subsubsection{Etapa III - Generalización del MDE}

En la tercera etapa se aplica la generalización condicionada por puntos críticos. Primeramente, las mallas de los MDE original y generalizado son proyectadas al plano horizontal. Sobre este plano horizontal es definida una región de Voronoi para cada punto de la malla del MDE generalizado. Esta región es construida a partir de los vértices de cada elemento de malla del MDE original que contiene un vértice del MDE generalizado (Figura 2). Si al menos uno de estos puntos del MDE original posee un valor de elevación crítico máximo o mínimo, se impone, como valor de elevación para el punto del MDE generalizado, el correspondiente al punto crítico del MDE original que posea el mayor valor de ranking. En caso que ninguno de los puntos involucrados del MDE original posea un valor crítico de elevación, el valor impuesto corresponderá al valor estimado por medio de una interpolación bilineal. Debido al tipo de estructura del MDE, en su proyección horizontal, la región de Voronoi resultante es un cuadrado de iguales dimensiones a la resolución espacial del MDE original y la aplicación del 
modelo de interpolación, se reduce a la media aritmética de los valores de elevación de los cuatro vértices del elemento de malla involucrado en el MDE original. El modelo de interpolación bilineal fue elegido para esta operación debido a que, dentro de los modelos isomórficos aplicados a este tipo de datos (HU et al., 2009), este modelo presenta el mejor desempeño en cuanto a su simplicidad de cálculo.

Figura 2 - Interpolación bilineal del valor de elevación para un punto del MDE generalizado.

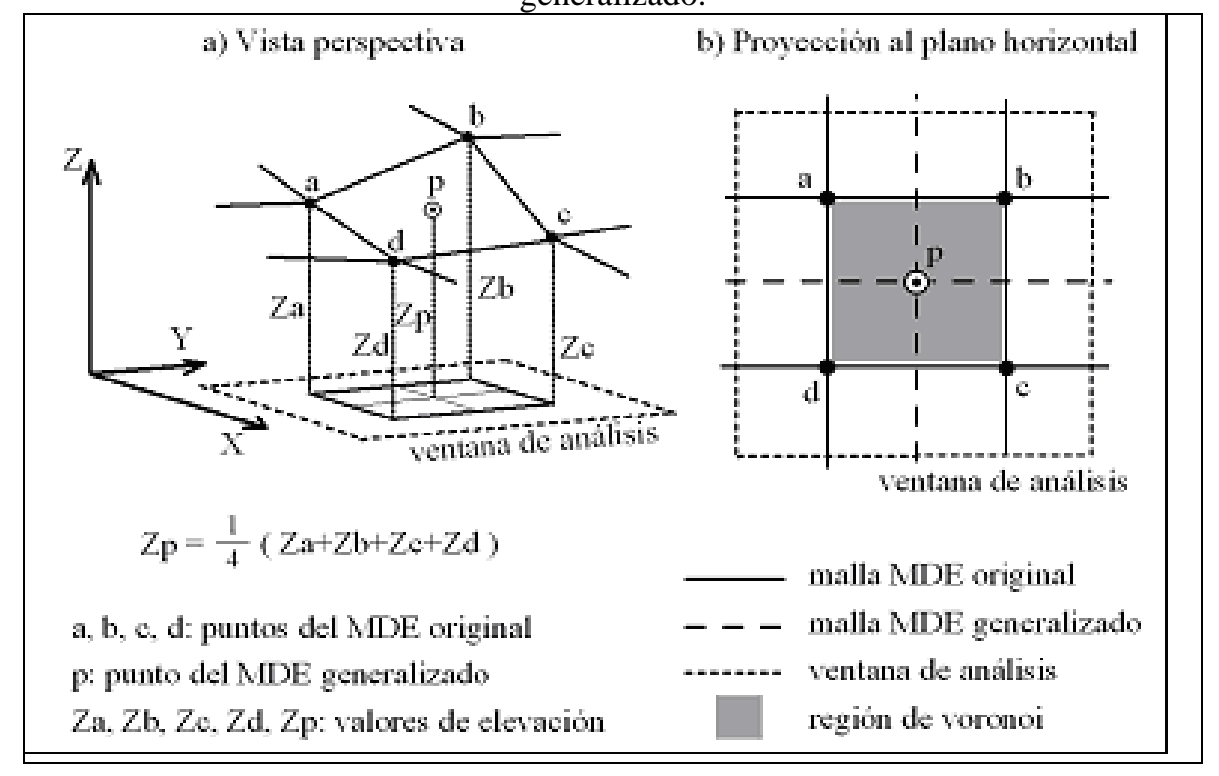

\subsection{Implementación de Algoritmos}

Los datos de elevación fueron tratados en forma de matrices de elevación, tal que las líneas ortogonales que componen la malla regular de un MDE son consideradas como filas y columnas en el sistema matricial. En este sistema cada punto es representado por una celda de iguales dimensiones del elemento de malla, y cuyo centro coincide con la posición horizontal del punto.

Los algorítmos computacionales que permitieron el montaje de los procesos de generalización se construyeron en el lenguaje de programación GNU Octave. Para la identificación de puntos con valores críticos correspondientes a cimas y crestas se utilizó el método de PEUCKER y DOUGLAS (1975), disponible en la librería de análisis geoespacial Sextante, incluido en la versión 1.10 del software gvSIG (rutina Clasificación de formas de terreno). Este algoritmo identifica siete situaciones críticas para un punto respecto a sus vecinos en una ventana de análisis de 3 por 3 celdas: cima, depresión, paso, línea de ruptura convexa, línea de ruptura cóncava, cresta y canal. En 
los experimentos de generalización se utilizó solamente los valores que denotan máximos de elevación, es decir, cimas y crestas.

Figura 3 - Diagrama de flujo del método de generalización condicionada por puntos críticos de terreno.

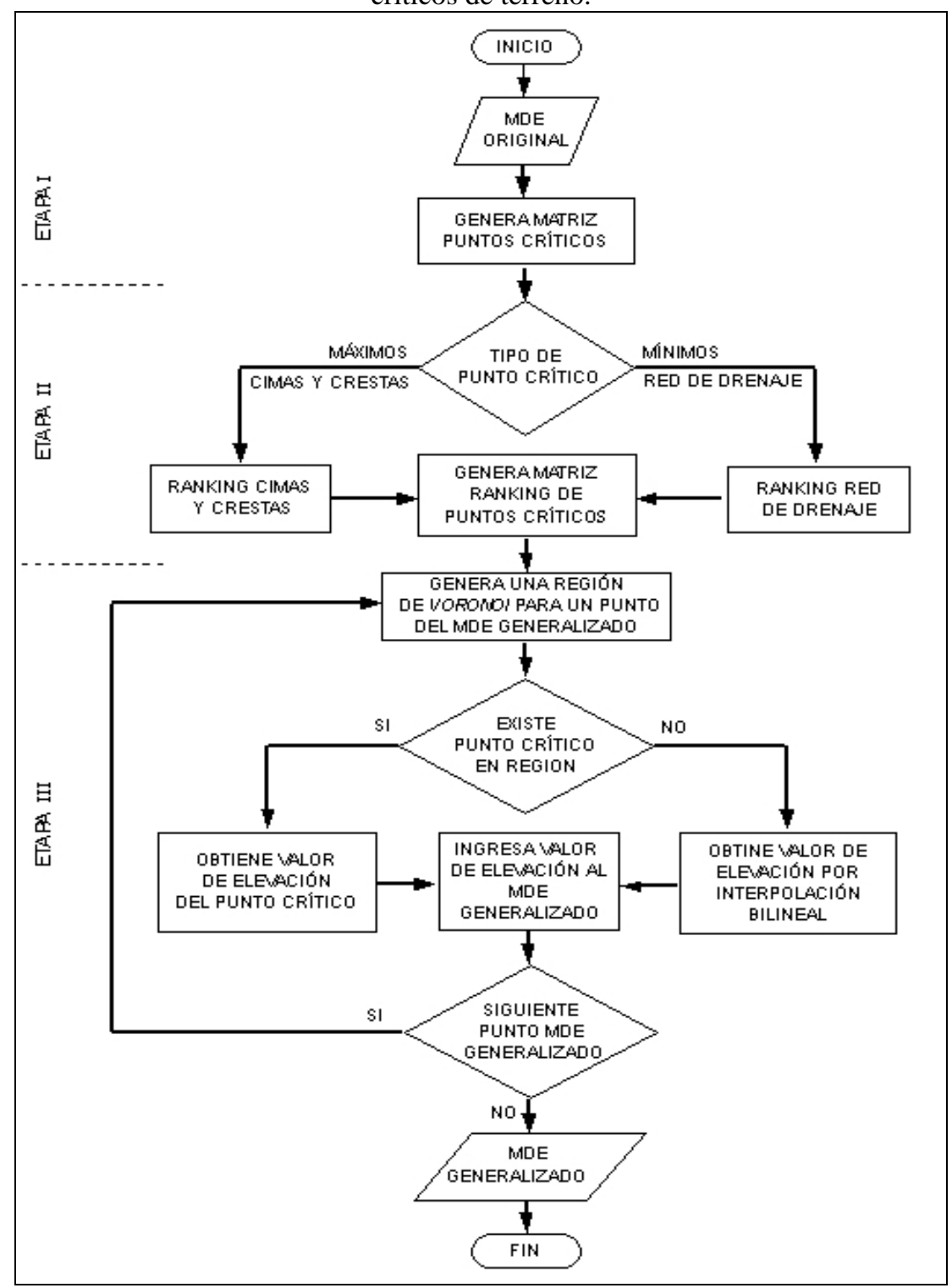

Bol. Ciênc. Geod., sec. Artigos, Curitiba, v. 17, nº 3, p.439-457, jul-set, 2011. 
La identificación de puntos que describen una red de drenaje, se consiguió con la aplicación de una modificación del método descrito por JENSON y DOMINGUE (1988), disponible en el software Idrisi32 (rutina RUNOFF). Este algoritmo calcula la acumulación de lluvia por unidad de celda en función del valor de elevación de cada celda, asignándole a cada celda un valor igual al número de celdas que fluyen hacia esta. Así, por ejemplo, una celda con valor de acumulación de flujo igual a $n$, implica que el flujo de lluvia que recibe la celda corresponde a la acumulación de lluvia de $n$ celdas. Dado que este software trabaja con formato de escritura de archivo rst, la conversión de formatos se realizó con la biblioteca de algorítmos de conversión $G D A L$, disponible en el software GRASS 6.4.0svn (2009). Finalmente, el software GS+ Geostatistics for the Environmental Sciences version 5.1.1 fue utilizado para calcular los variogramas isotrópicos en el análisis comparativo del comportamiento espacial de los valores de elevación en cada MDE generalizado.

Los experimentos fueron montados en una máquina tipo PC, con procesador Intel Atom de $1.60 \mathrm{GHz}$ y $1 \mathrm{~GB}$ de memoria RAM. Los tiempos de procesamiento para los algoritmos utilizados para generalizar el MDE con mayor cantidad de puntos (MDE 90 con 1.440.000 puntos) fueron los siguientes: identificación puntos críticos máximos 10 segundos, identificación puntos críticos mínimos 21 segundos, matriz de ranking de puntos críticos 0,6 segundos y MDE generalizado 187 segundos. Dado que los tiempos de procesamiento no son excesivos, y estos en la práctica son independientes de los valores contenidos en las matrices, se estimó innecesario realizar una selección de puntos críticos para reducir los tiempos de procesamiento.

\section{EXPERIMENTOS}

\subsection{Generalización de MDE Condicionada a Puntos Críticos}

Los experimentos consideraron cuatro generalizaciones a partir del MDE original de 90m de resolución espacial (MDE 90): MDE 180, MDE 360, MDE 720 y MDE1440, de 180m, 360m, 720m y 1440m de resolución espacial respectivamente.

Con la aplicación del algorítmo de PEUCKER y DOUGLAS (1975) se obtuvo una matriz que identifica diversas situaciones críticas. A esta matriz le fue aplicada un filtro de manera de obtener una matriz con la identificación solamente de los puntos con elevaciones críticas máximas, que denoten una cima o una cresta. En forma independiente, se aplicó el algoritmo que determina la acumulación de flujo por celda, de lo cual se obtuvo una matriz con los valores de acumulación de flujo por cada celda. Esta matriz fue filtrada aplicando un valor umbral de inicio de flujo, el cual define el valor de acumulación mínimo para considerar el inicio de una red de drenaje. Aplicado el filtro se obtuvo una matriz con la identificación de los puntos con valores críticos mínimos. Los valores para el umbral, correspondiente a la definición del inicio de red de drenaje (inicio de flujo), fueron determinados según el siguiente criterio: se estimó un flujo máximo para una ventana de 2 por 2 celdas en el MDE 1440, que corresponde al 
valor 3, luego se estimó, su equivalencia en cada una de las otras resoluciones de MDE (Tabla 1).

Identificadas las celdas que contienen valores críticos máximos y mínimos, fue aplicado el proceso de ranking que permite ponderar la importancia de cada valor de elevación en su contexto de vecindad. Se asignó un valor de ranking proporcional al valor de elevación (Figura 4): 100\% para celdas con valor de elevación de 4.074m, que corresponde al valor de elevación máxima en la zona del MDE; y de $0 \%$ para celdas con valor de elevación 113m, correspondiente al valor de elevación mínima del MDE. Para las celdas con valores críticos correspondientes a acumulación de flujo se aplicó un ranking inversamente proporcional al valor de elevación, es decir, 0\% para celdas con valor de elevación de 4.071m y 100\% para celdas con valor de elevación igual a 113m. De esta forma, se generó una matriz de ponderación (matriz de ranking) que intervino en la etapa de estimación del valor de elevación para cada punto del MDE generalizado.

Tabla 1 - Umbral para inicio de acumulación de flujo para cada MDE.

\begin{tabular}{c|c|c|c}
\hline MDE & Ventana & Total de celdas & Umbral \\
\hline MDE 90 & $25 \times 25$ & 625 & 624 \\
\hline MDE 180 & $13 \times 13$ & 169 & 168 \\
\hline MDE 360 & $7 \times 7$ & 49 & 48 \\
\hline MDE 720 & $4 \times 4$ & 16 & 15 \\
\hline MDE 1440 & $2 \times 2$ & 4 & 3 \\
\hline
\end{tabular}

Figura 4 - Ranking de ponderación para valores críticos de terreno.

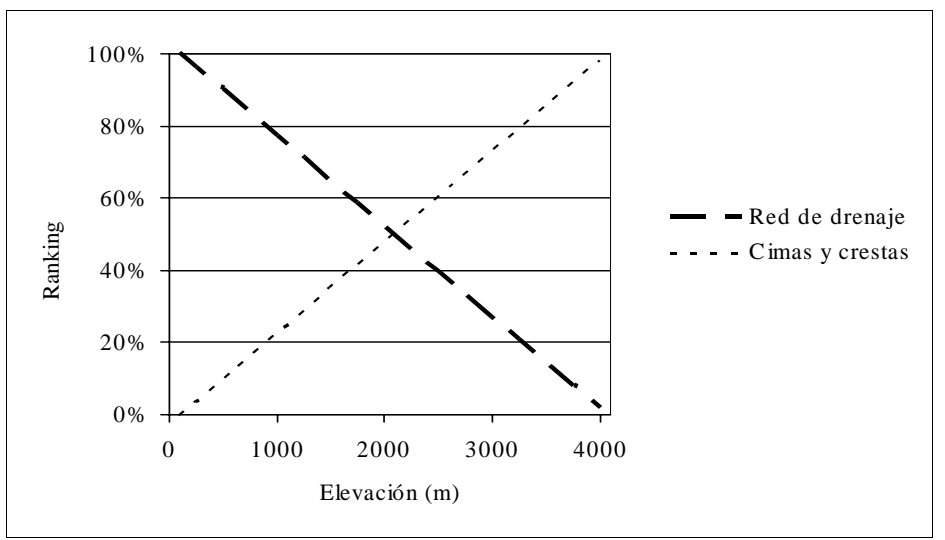

Bol. Ciênc. Geod., sec. Artigos, Curitiba, v. 17, nํ 3, p.439-457, jul-set, 2011. 


\subsection{Evaluación}

Paralelamente y a modo de comparación, se generaron MDE generalizados en las mismas resoluciones pero su construcción no fue condicionada por puntos críticos de terreno. El valor de elevación para cada punto del MDE generalizado fue obtenido por la sola aplicación de la media aritmética, a partir de los valores de elevación del MDE original. Los MDE generalizados por este método son identificados con la sigla MA y los obtenidos por aplicación del método condicionado por puntos críticos por la sigla PC.

Figura 5 - Red de drenaje evaluada em las MDE y cimas evaluadas em MDE1440.

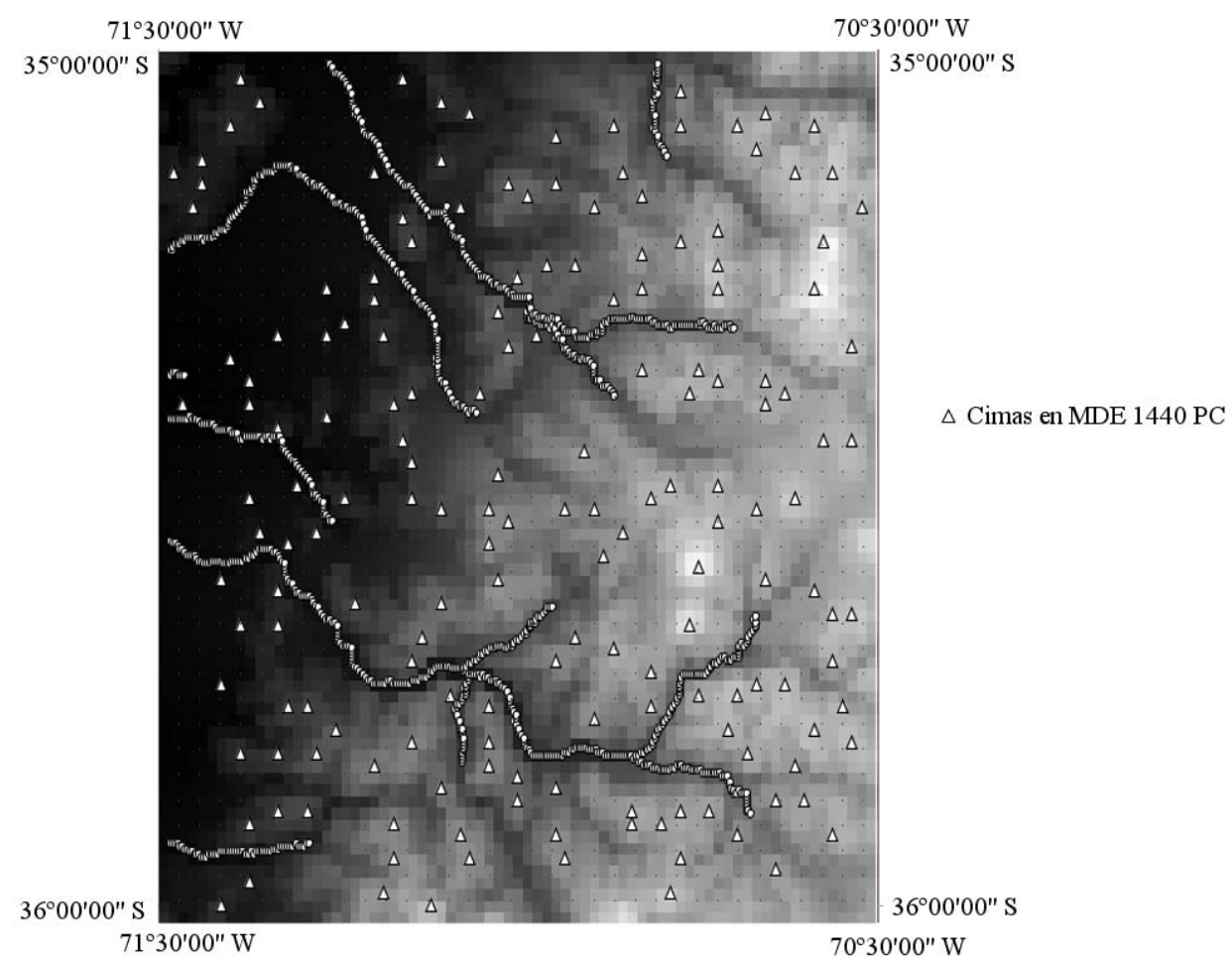

La evaluación consistió en comparar, entre las generalizaciones de MDE condicionados y no condicionados por puntos críticos de terreno, la cantidad de puntos con valores de elevación críticos máximos y mínimos que se pueden obtener en cada MDE, como así también sus valores extremos de elevación. Seguidamente, se evaluaron las diferencias absolutas horizontales, verticales y tridimensionales de cimas y de la red de drenaje obtenida en los MDE generalizados, cuando son comparados con los obtenidos en el MDE original (Figura 5). Dado que en cada MDE generalizado la 
red de drenaje presenta diferentes niveles de detalle, por ejemplo, cursos de agua menores no aparecen en resoluciones más gruesas de un MDE, se optó, para efectos de evaluación, considerar el trazado de la red de drenaje que puede ser identificado en todas las resoluciones de los MDE.

Figura 6 - (a) Superficies original, (b) superficie ajustada y (c) sustracción de superficies.

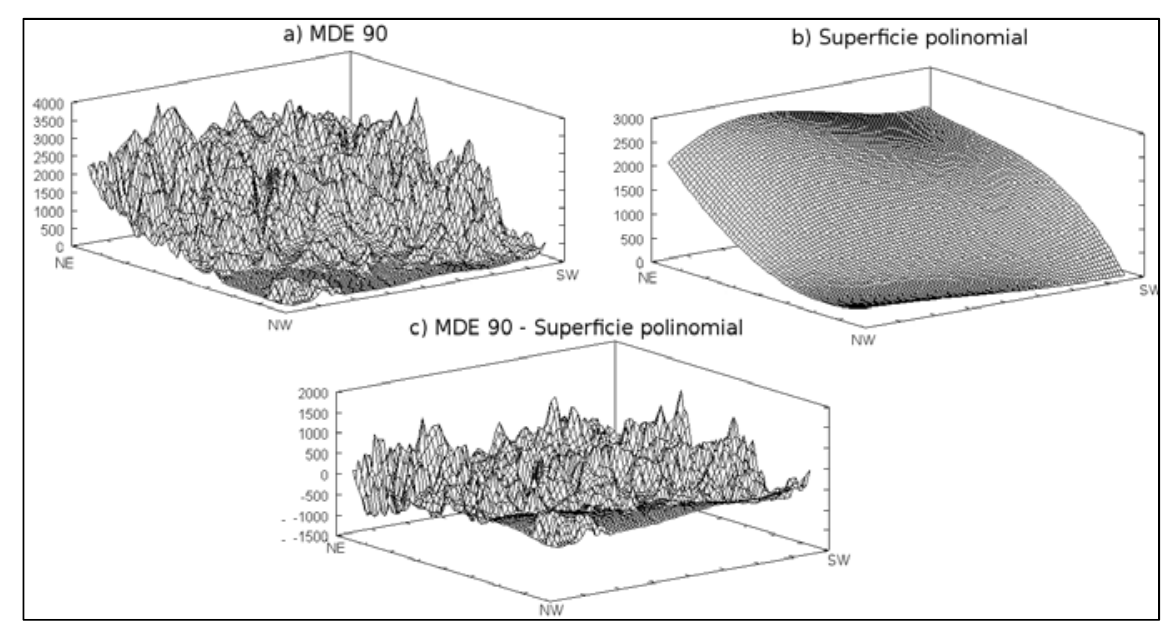

Finalmente, variogramas isotrópicos fueron creados para analizar comparativamente el comportamiento espacial de los valores de elevación en cada MDE generalizado, a través de una muestra de 5.625 puntos para cada MDE, obtenidos sistemáticamente y considerando cada punto de elemento de malla para el MDE 1440. Debido a que los valores de elevación presentaron característica no estacionaria, fue ajustada una superficie polinomial a los 5.625 puntos de muestreo sobre el MDE 90. Teniendo como base esta nueva superficie, fueron obtenidos los nuevos valores de elevaciones, que corresponden a las diferencias de elevación entre los valores del MDE y los valores calculados sobre la superficie polinomial (Figura 6). De esta manera, las elevaciones se tornaron una variable regionalizada estacionaria, susceptible de ser analizada mediante variogramas isotrópicos. El modelo polinomial de tercer grado usado es dado por la siguiente ecuación:

$$
\begin{aligned}
Z=a_{0}+a_{1} x+a_{2} x^{2} & +a_{3} x^{3}+a_{4} y+a_{5} x y+a_{6} x^{2} y+a_{7} x^{3} y+a_{8} y^{2}+a_{9} x y^{2}+a_{10} x^{2} y^{2} \\
& +a_{11} x^{3} y^{2}+a_{12} y^{3}+a_{13} x y^{3}+a_{14} x^{2} y^{3}+a_{15} x^{3} y^{3}
\end{aligned}
$$

donde $Z$ es el valor de elevación en la superficie polinomial ajustada, $a_{0}, a_{1}, \ldots, a_{15}$ son los parámetros calculados $y, x$ e $y$ las coordenadas cartesianas del punto. 


\section{RESULTADOS}

En el MDE original (MDE 90) se identificaron, de un total de 1.440.000 puntos, 46.681 puntos con valores críticos máximos correspondientes a cimas, 350.734 correspondientes a crestas y 45.100 puntos correspondientes a red de drenaje. El valor de elevación máximo para cima corresponde a $4.075 \mathrm{~m}$ y el valor de elevación mínimo para red de drenaje es $118 \mathrm{~m}$. Se observa que este último valor no corresponde al valor mínimo de elevación del MDE 90, a saber 113m, esto se debe al desempeño del algorítmo que calcula la acumulación de flujo, el cual excluye las celdas de borde del MDE. La totalidad de los puntos identificados como cima, crestas y red de drenaje fueron utilizados para construir la matriz de ranking de puntos críticos. Finalmente, aplicado el método de generalización condicionada por puntos críticos se obtuvieron MDE generalizados en las resoluciones 180m, 360m, 720m y 1440m.

\subsection{Evaluación de Valores Extremos en Cada MDE}

La generalización de MDE condicionada por puntos críticos mantuvo inalterados los valores máximos de elevación correspondientes a cimas y a crestas, salvo el caso de crestas en el MDE 1440 PC que presenta una diferencia de $-22 \mathrm{~m}$ en relación al valor original (Tabla 2). Esta situación puede ser explicada por el hecho que en el proceso de generalización, crestas vecinas a una cima con mayor valor de elevación obtienen valores de ranking menores al de la cima, y por tanto, en el proceso de clasificación de puntos críticos para el nuevo MDE generalizado, estas crestas no clasifican como punto crítico ha preservar. Para el caso de la red de drenaje, los MDE PC presentaron todos un mismo valor de elevación mínima, que corresponde al punto más bajo de la red de drenaje. Valores máximos de elevación para la red de drenaje presentan diferencias con tendencia al alza en su valor. Esto puede explicarse por el criterio usado en el ranking de ponderación de los puntos críticos, dando mayor ponderación a cimas y crestas y menor ponderación a la red de drenaje en zonas de elevaciones mayores. Así los inicios de flujo aparecen con valores de elevación mayor en cada generalización.

En la generalización por media aritmética, los mismos rasgos evaluados presentan diferencias en sus valores extremos. En general, disminuyen los valores de elevación máximos y aumentan los valores de elevación mínimos en cada generalización. La cantidad de celdas con valores críticos también disminuye respecto a la generalización condicionada por puntos críticos. Al existir menor diferencia entre los valores máximos y mínimos, la superficie descrita en los MDE MA se presenta como una superficie achatada respecto de la superficie original. 
Tabla 2 - Valores extremos en cada MDE.

\begin{tabular}{|c|c|c|c|c|c|c|}
\hline \multirow[b]{2}{*}{ Rasgo } & \multirow{2}{*}{ Ítem } & \multirow{2}{*}{$\begin{array}{l}\text { Original } \\
\text { MDE } 90\end{array}$} & \multicolumn{4}{|c|}{$\begin{array}{l}\text { Generalización condicionada por puntos } \\
\text { críticos }\end{array}$} \\
\hline & & & $\begin{array}{l}\text { MDE } \\
\text { 180PC }\end{array}$ & $\begin{array}{l}\text { MDE } \\
\text { 360PC }\end{array}$ & $\begin{array}{l}\text { MDE } \\
\text { 720PC }\end{array}$ & $\begin{array}{l}\text { MDE } \\
\text { 1440PC }\end{array}$ \\
\hline \multirow{3}{*}{ Cimas } & Elevación máxima & $4.075,00$ & $4.075,00$ & $4.075,00$ & $4.075,00$ & $4.075,00$ \\
\hline & Elevación mínima & 120,00 & 120,00 & 126,00 & 131,00 & 178,00 \\
\hline & Total de celdas & 46.681 & 9.347 & 1.923 & 523 & 172 \\
\hline \multirow{3}{*}{ Crestas } & Elevación máxima & $4.074,00$ & $4.074,00$ & $4.074,00$ & $4.074,00$ & $4.052,00$ \\
\hline & Elevación mínima & 119,00 & 120,00 & 122,00 & 124,00 & 161,00 \\
\hline & Total de celdas & 350.734 & 123.535 & 30.364 & 7.081 & 1.746 \\
\hline \multirow{4}{*}{$\begin{array}{l}\text { Red de } \\
\text { drenaje }\end{array}$} & Elevación máxima & $3.001,00$ & $3.124,00$ & $3.336,00$ & $3.382,00$ & $3.462,00$ \\
\hline & Elevación mínima & 118,00 & 118,00 & 118,00 & 118,00 & 118,00 \\
\hline & Acumulación de flujo máximo & 1.168 & 584 & 292 & 146 & 73 \\
\hline & Total de celdas & 45.100 & 22.705 & 10.278 & 4.188 & 1.890 \\
\hline \multirow[b]{2}{*}{ Rasgo } & \multirow[b]{2}{*}{ Ítem } & Original & \multicolumn{4}{|c|}{ Generalización por media aritmética } \\
\hline & & MDE 90 & $\begin{array}{l}\text { MDE } \\
\text { 180MA }\end{array}$ & $\begin{array}{l}\text { MDE } \\
\text { 360MA }\end{array}$ & $\begin{array}{c}\text { MDE } \\
\text { 720MA }\end{array}$ & $\begin{array}{l}\text { MDE } \\
\text { 1440MA }\end{array}$ \\
\hline \multirow{3}{*}{ Cimas } & Elevación máxima & $4.075,00$ & $4.051,25$ & $4.000,81$ & $3.911,34$ & $3.688,78$ \\
\hline & Elevación mínima & 120,00 & 121,75 & 126,81 & 163,61 & 157,04 \\
\hline & Total de celdas & 46.681 & 5.985 & 1.393 & 444 & 106 \\
\hline \multirow{3}{*}{ Crestas } & Elevación máxima & $4.074,00$ & $4.051,00$ & $3.981,06$ & $3.890,80$ & $3.688,21$ \\
\hline & Elevación mínima & 119,00 & 119,00 & 121,69 & 123,97 & 122,87 \\
\hline & Total de celdas & 350.734 & 96.101 & 24.837 & 6.178 & 1.531 \\
\hline \multirow{4}{*}{$\begin{array}{l}\text { Red de } \\
\text { drenaje }\end{array}$} & Elevación máxima & $3.001,00$ & $3.312,25$ & $3.291,50$ & $2.982,11$ & $3.092,46$ \\
\hline & Elevación mínima & 118,00 & 118,00 & 119,13 & 120,42 & 122,87 \\
\hline & Acumulación de flujo máximo & 1.168 & 584 & 292 & 146 & 73 \\
\hline & Total de celdas & 45.100 & 20.802 & 9.339 & 3.998 & 2.003 \\
\hline
\end{tabular}

\subsection{Evaluación de Diferencias Absolutas Horizontales, Verticales y} Tridimensionales de los Rasgos Cimas y red de Drenaje

En la evaluación de rasgos del terreno fueron consideradas las cimas y la red de drenaje. Estos rasgos fueron obtenidos en cada MDE generalizado y luego comparados con los obtenidos en el MDE original (Tabla 3, Tabla 4 y Tabla 5). En la evaluación del 
rasgo red de drenaje, se observa que, tanto para las diferencias absolutas horizontales y las tridimensionales, los valores de las medias resultaron menores para los MDE generalizados por puntos críticos. Al evaluar las medias más dos desviaciones estándar, para una probabilidad del 95\%, estas presentaron valores menores para la generalización condicionada por puntos críticos y próximas a la resolución del MDE respectivo, en cambio para las generalizaciones por media aritmética, estas presentaron valores notoriamente mayores a la resolución del MDE respectivo. Destaca el caso del MDE 1440 para el cual la media más dos desviaciones estándar fue 2,5 veces la resolución del MDE. No obstante lo anterior, las medias y desviaciones estándar para las diferencias verticales absolutas, presentan valores notoriamente menores en los MDE generalizados por media aritmética.

Tabla 3 - Diferencias absolutas horizontales.

\begin{tabular}{c|c|c|c|c|c|c|c|c}
\hline \multirow{2}{*}{ MDE } & \multicolumn{2}{c|}{ Diferencias absolutas horizontales } & \multicolumn{3}{c}{ Diferencias absolutas horizontales } \\
\cline { 2 - 9 } & $\begin{array}{c}\text { Cantida } \\
\text { d de } \\
\text { celdas }\end{array}$ & Media & $\begin{array}{c}\text { Desviaci } \\
\text { ón } \\
\text { estándar }\end{array}$ & $\begin{array}{c}\text { Media }+ \\
\text { 2 desv. } \\
\text { estándar }\end{array}$ & $\begin{array}{c}\text { Cantidad } \\
\text { de celdas }\end{array}$ & Media & $\begin{array}{c}\text { Desviación } \\
\text { estándar }\end{array}$ & $\begin{array}{c}\text { Media + } \\
\text { 2 desv. } \\
\text { estándar }\end{array}$ \\
\hline 180PC & 2233 & 76,75 & 53,43 & 183,61 & 9347 & 64,33 & 23,19 & 110,71 \\
180MA & 2103 & 101,38 & 139,46 & 380,30 & 5985 & 75,96 & 53,66 & 183,28 \\
\hline 360PC & 1054 & 138,83 & 151,89 & 442,60 & 1923 & 121,60 & 47,57 & 216,74 \\
360MA & 1010 & 184,93 & 303,61 & 792,15 & 1393 & 126,85 & 73,61 & 274,08 \\
\hline $720 P C$ & 492 & 220,06 & 184,55 & 589,16 & 523 & 228,91 & 100,30 & 429,50 \\
$720 M A$ & 498 & 274,84 & 326,85 & 928,53 & 444 & 227,80 & 112,60 & 453,01 \\
\hline 1440PC & 245 & 403,31 & 294,95 & 993,21 & 172 & 380,38 & 198,23 & 776,85 \\
$1440 M A$ & 242 & 684,07 & $1.421,54$ & $3.527,15$ & 106 & 394,72 & 199,91 & 794,55 \\
\hline
\end{tabular}

En cuanto al rasgo cimas, las magnitudes de media y desviación estándar para diferencias absolutas horizontales y tridimensionales, presentaron valores menores a los presentados para la red de drenaje. Evaluados a dos desviaciones estándar de la media, presentaron magnitudes inferiores a la resolución del MDE, tanto en la generalización por media aritmética como en la generalización condicionada por puntos críticos. La única excepción es el MDE 180 MA que presentó un valor ligeramente sobre la resolución del MDE. En cuanto a las diferencias absolutas verticales, sus respectivas medias más dos desviaciones estándar resultaron menores para la generalización condicionada por puntos críticos que para los MDE generalizados por media aritmética. 
Tabla 4 - Diferencias absolutas verticales.

\begin{tabular}{c|c|c|c|c|c|c|c|c}
\hline \multirow{2}{*}{ MDE } & \multicolumn{3}{|c|}{ Diferencias absolutas verticales } & \multicolumn{3}{c}{ Diferencias absolutas verticales } \\
\cline { 2 - 9 } & $\begin{array}{c}\text { Cantidad } \\
\text { de celdas }\end{array}$ & Media & $\begin{array}{c}\text { Desviación } \\
\text { estándar }\end{array}$ & $\begin{array}{c}\text { Media }+ \\
\text { 2 desv. } \\
\text { estándar }\end{array}$ & $\begin{array}{c}\text { Cantidad } \\
\text { de celdas }\end{array}$ & $\begin{array}{c}\text { Desviac } \\
\text { ión } \\
\text { estánda } \\
\text { r }\end{array}$ & $\begin{array}{c}\text { Media + } \\
\text { 2 desv. } \\
\text { estándar }\end{array}$ \\
\hline 180PC & 2233 & 1,47 & 6,38 & 14,24 & 9347 & 0,05 & 2,54 & 5,14 \\
180MA & 2103 & 2,97 & 5,65 & 14,27 & 5985 & 7,70 & 20,73 & 49,17 \\
\hline 360PC & 1054 & 12,62 & 29,79 & 72,20 & 1923 & 3,32 & 42,89 & 89,10 \\
360MA & 1010 & 9,58 & 13,98 & 37,55 & 1393 & 33,73 & 41,89 & 117,51 \\
\hline $720 P C$ & 492 & 47,22 & 84,58 & 216,37 & 523 & 13,88 & 67,40 & 148,68 \\
$720 M A$ & 498 & 23,37 & 28,36 & 80,09 & 444 & 82,14 & 48,68 & 179,50 \\
\hline 1440PC & 245 & 144,24 & 204,81 & 553,85 & 172 & 64,63 & 143,02 & 350,67 \\
1440MA & 242 & 57,74 & 60,73 & 179,20 & 106 & 154,31 & 100,59 & 355,48 \\
\hline
\end{tabular}

Tabla 5 - Diferencias absolutas tridimensionales.

\begin{tabular}{|c|c|c|c|c|c|c|c|c|}
\hline \multirow[b]{2}{*}{ MDE } & \multicolumn{4}{|c|}{ Diferencias absolutas tridimensionales } & \multicolumn{4}{|c|}{ Diferencias absolutas tridimensionales } \\
\hline & $\begin{array}{l}\text { Cantidad } \\
\text { de celdas }\end{array}$ & Media & $\begin{array}{c}\text { Desviació } \\
\text { n } \\
\text { estándar }\end{array}$ & $\begin{array}{c}\text { Media + } \\
2 \text { desv. } \\
\text { estánda } \\
\text { r }\end{array}$ & $\begin{array}{c}\text { Cantida } \\
\text { d de } \\
\text { celdas }\end{array}$ & Media & $\begin{array}{c}\text { Desviaci } \\
\text { ón } \\
\text { estánda } \\
\text { r }\end{array}$ & $\begin{array}{c}\text { Media + } \\
2 \text { desv. } \\
\text { estánda } \\
\text { r }\end{array}$ \\
\hline 180PC & 2233 & 76,93 & 53,57 & 184,07 & 9347 & 64,33 & 23,32 & 110,98 \\
\hline 180MA & 2103 & 101,60 & 139,44 & 380,49 & 5985 & 77,20 & 56,38 & 189,96 \\
\hline $360 P C$ & 1054 & 141,99 & 152,41 & 446,80 & 1923 & 123,93 & 59,52 & 242,96 \\
\hline 360MA & 1010 & 186,25 & 303,28 & 792,80 & 1393 & 134,26 & 79,85 & 293,96 \\
\hline 720PC & 492 & 239,04 & 186,32 & 611,68 & 523 & 235,01 & 109,36 & 453,73 \\
\hline $720 \mathrm{MA}$ & 498 & 279,03 & 325,36 & 929,74 & 444 & 247,77 & 110,88 & 469,53 \\
\hline 1440PC & 245 & 471,53 & 299,84 & $1.071,21$ & 172 & 405,87 & 209,27 & 824,40 \\
\hline 1440MA & 242 & 695,29 & $1.418,54$ & $3.532,38$ & 106 & 434,41 & 202,25 & 838,90 \\
\hline
\end{tabular}

\subsection{Evaluación por Medio de Variogramas Isotrópicos}

A partir de una muestra sistemática de 5.625 puntos, espaciados a $1440 \mathrm{~m}$ sobre la zona de prueba, variogramas experimentales isotrópicos fueron construidos para el análisis de la varianza espacializada de los valores de elevación (Figura 7). 
Figura 7 - Variogramas Isotrópicos MDE 90, MDE 180, MDE 360, MDE 720 y MDE 1440 .

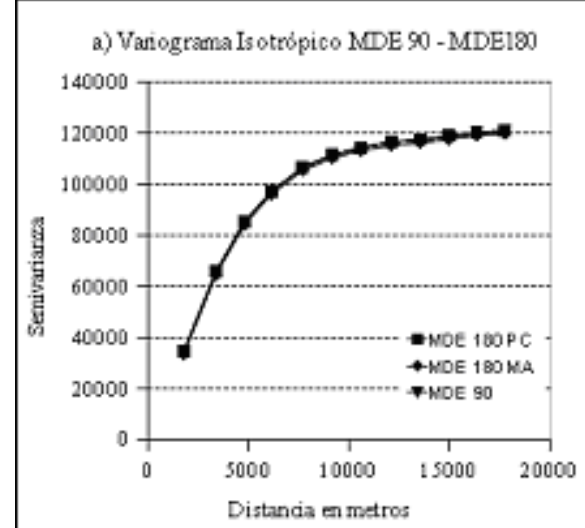

c) Variograma Is otrópico $\mathrm{MDE} 90-\mathrm{MDE} 720$

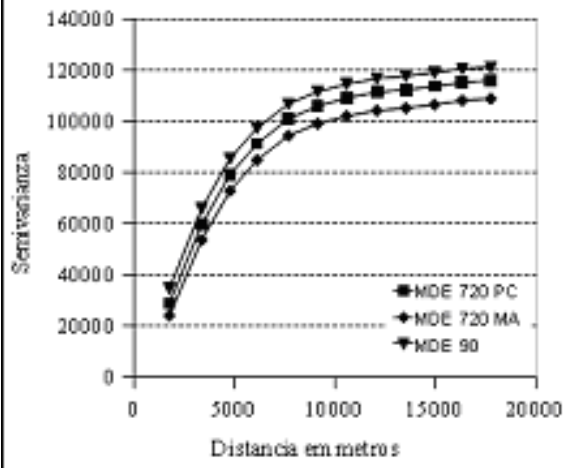

b) Variograma Isotrópico MDE90 - MDE 360

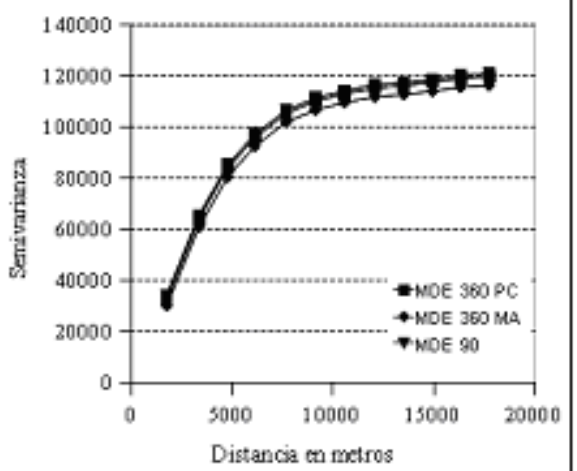

d) Variograma Is otrópico MDE $90-M D E 1440$

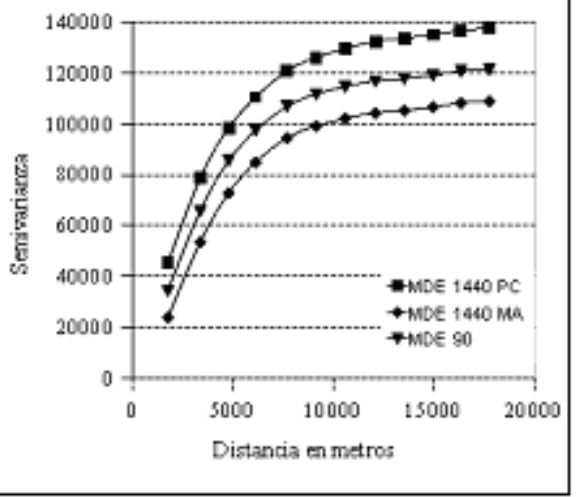

Los variogramas evidencian que los residuos mantienen una correlación espacial a distancias inferiores a los $6 \mathrm{~km}$. Para los MDE 180 PC y MDE 180 MA no presentan diferencias significativas en sus varianzas respecto al MDE 90. Los variogramas para los MDE generalizados por media aritmética evidencian una disminución de las varianzas a medida que los MDE son generalizados a resoluciones más gruesas. Esto tiene un efecto de suavizamiento en la representación de la superficie. Los MDE generalizados por condicionamiento de puntos críticos presentan varianzas más cercanas a las varianzas del MDE original, salvo en el MDE 1440 donde las varianzas son claramente mayores. Este comportamiento evidencia que los MDE 180, MDE 360 y MDE 720 cuyas generalizaciones son condicionadas por puntos críticos de terreno tienen un mejor desempeño que los modelos generalizados por media aritmética, en

Bol. Ciênc. Geod., sec. Artigos, Curitiba, v. 17, no 3, p.439-457, jul-set, 2011. 
cuanto a preservar el comportamiento de las variaciones de los valores de elevación. Para el caso del MDE 1440 el variograma evidencia un efecto de exageración de las variaciones de elevación, por el hecho de que la muestra de puntos corresponde a los puntos de centros de celda del MDE 1440 y por tanto corresponde al $100 \%$ de los valores del MDE; por otro lado, los valores de elevación contenidos corresponden a los valores máximos y mínimos presentes en la zona de prueba.

\section{CONCLUSIÓN}

Los experimentos realizados en este estudio abordaron la implementación y evaluación del método de generalización de MDE condicionada por puntos críticos de terreno, el cual se compone de tres etapas. En la primera etapa son seleccionadas los puntos del MDE con valores críticos de elevación que caracterizan los rasgos del terreno. En la segunda etapa, se genera una matriz de ranking que pondera la importancia de cada punto crítico según su valor de elevación y rasgo representado. En la tercera etapa se aplica la generalización condicionada por puntos los críticos, tal que si en la región circundante al punto, cuyo valor de elevación se quiere estimar, existe un punto con valor de elevación crítico máximo o mínimo, el valor de elevación de este punto es impuesto como valor de elevación para el MDE generalizado. En caso de no existir puntos críticos en la región, entonces se aplica una interpolación bilinear. A modo de comparación, se realizaron generalizaciones en las mismas resoluciones espaciales pero utilizando solamente la media aritmética para el cambio de resolución. Los resultados evidenciaron que al aplicar una generalización condicionada por puntos críticos las posiciones horizontales de la red de drenaje evaluada en general fueron preservadas en las resoluciones de los MDE respectivos. También se observó que valores de elevaciones máximas y de acumulación de flujo se mantuvieron inalterados en sus respectivas resoluciones para los MDE, y el valor de las varianzas espacializadas tienden a mantener el valor para generalizaciones próximas a la resolución espacial del DEM original. Al disminuir el nivel de resolución en 4 veces, el valor de las varianzas espacializadas aumenta evidenciando un efecto inverso al de suavizamiento.

Por otro lado, la aplicación de generalización por media aritmética tiene un efecto de achatamiento y de suavizamiento del MDE generalizado y no necesariamente mantiene los rasgos que caracterizan el terreno dentro de la resolución del MDE generalizado. En la red de drenaje evaluada, los desplazamientos aparentes de la red fueron mayores a la resolución del MDE, no obstante que las diferencias de elevación hayan sido menores.

Dado lo anterior, y sobre la base de los experimentos realizados, se puede concluir que el método de generalización condicionada por puntos críticos presenta mejor desempeño que la generalización por media aritmética, y que es un método viable cuando se busca preservar rasgos que caracterizan el terreno. Por otra parte, se constató que el análisis de diferencias de elevación por si solo no garantiza una adecuada validación de un método de generalización de MDE, lo cual quedó evidenciado cuando 
valores menores de diferencias de elevación ocurrieron en puntos con mayores diferencias de posición horizontal.

\section{REFERENCIAS BIBLIOGRÁFICAS}

AI, T., 2007. The drainage network extraction from contour lines for contour line generalization. ISPRS Journal of Photogrammetry and Remote Sensing, n. 62, p. 93103.

AI, T., LI, J., 2010. A DEM generalization by minor valley branch detection and grid filling. ISPRS Journal of Photogrammetry and Remote Sensing, n. 65, p. 198-207.

BURROUGH, P.A., MCDONNELL, R., 1998. Principles of Geographical Information Systems, Spatial Information Systems and Geostatistics, 2a Edición, Oxford University Press. p.333.

FARR, T. G., P. A. ROSEN, E. CARO, R. CRIPPEN, R. DUREN, S. HENSLEY, M. KOBRICK, M. PALLER, E. RODRIGUEZ, L. ROTH, D. SEAL, S. SHAFFER, J. SHIMADA, J. UMLAND, M. WERNER, M. OSKIN, D. BURBANK, D. ALSDORF, 2007. The Shuttle Radar Topography Mission, Reviews of Geophysics, 45, RG2004, doi:10.1029/2005RG000183. Disponible en: http:// www.agu.org/pubs/crossref/2007/2005RG000183.shtml. Acceso en Ago. 2011.

FOWLER, R. J. AND LITTLE, J. J., 1979. Automatic Extraction of Irregular Network Digital Terrain Models, Computer Graphics, v. 13, n. 2, p. 199-207.

HERRINGTON, L., AND PELLEGRINI, G. 2000. An advanced shape of country classifier: extraction of surface features from DEMs. In Proceedings of the 4th International Conference on GIS and Environmental Modeling (GIS/EM4), Problems, Prospects and Research Needs, 2-8 September 2000, Banff, Alta. Disponible en http://www.colorado.edu/research/cires/banff/pubpapers/205/. Acceso en Mar. 2011.

HU, P.; LIU, X., 2009. On the accuracy of digital elevation models and their generalization. In $24^{\text {th }}$ International Cartographic Conference - ICC 2009, Santiago, Chile, Noviembre, 2009. Disponible en http://icaci.org/documents/ ICC_proceedings/ICC2009/html/refer/5_6.pdf. Acceso en Mar. 2011.

HU, P. LIU, X. AND HU, H., 2009. Isomorphism in digital elevation models and its implications to interpolation functions. Photogrammetric Engineering and Remote Sensing, n. 75, p. 713-722.

JENSON, S. K., DOMINGUE J. O., 1988. Extracting Topographic Structure from Digital Elevation Data for Geographic Information System Analysis, Photogrammetric Engineering and Remote Sensing, v. 54, n. 11, p. 1593-1600.

NIKOLAKOPOULOS, K.G., KAMARATAKIS, E.K., CHRYSOULAKIS N., 2006. SRTM vs ASTER elevation products. Comparison for two regions in Crete, Greece. International Journal of Remote Sensing, v. 27, n. 21, p. 4819-483. Disponible en http//.www.iacm.forth.gr/_docs/pubs/4/Nikolakopoulos_et_al_2006.pdf. Acceso en Mar. 2011 
PEUCKER, TH. K., DOUGLAS, D.H., 1975. Detection of surface-specific points by local parallel processing of discrete terrain elevation data, Comput. Vision, Graphics, Image Processing, v. 4, p. 375-387.

WEVER C., LINDEBERGER J., 1999. Experinces of 10 years laser scanning, p. 125132. Disponible en http://www.ifp.uni-stuttgart.de/publications/phowo99/ wever.pdf. Acceso en Mar. 2011.

ZHOU, Q., CHEN, Y., 2010. Generalization of DEM for terrain analysis using a compound method. ISPRS Journal of Photogrammetry and Remote Sensing, v. 66, n. 1, p. 38-45.

(Recebido em novembro de 2010. Aceito em setembro de 2011.) 